\title{
Clinical manifestations of device-related infective endocarditis in cardiac resynchronization therapy recipients
}

\author{
Ewa Jędrzejczyk-Patej ${ }^{1}$, Michał Mazurek ${ }^{1}$, Oskar Kowalski ${ }^{1}$, Adam Sokal ${ }^{1}$, Agnieszka Liberska ${ }^{1}$, \\ Mariola Szulik ${ }^{1}$, Tomasz Podolecki ${ }^{1}$, Zbigniew Kalarus ${ }^{2}$, Radosław Lenarczyk ${ }^{1}$
}

\author{
'Department of Cardiology, Congenital Heart Diseases and Electrotherapy, \\ Silesian Centre for Heart Diseases, Zabrze, Poland \\ 2Department of Cardiology, School of Medicine with the Division of Dentistry, Zabrze, \\ Poland
}

Submitted: 16 February 2018; Accepted: 4 April 2018;

Online publication: 7 August 2018

Arch Med Sci 2021; 17 (3): 638-645

DOI: https://doi.org/10.5114/aoms.2018.75893

Copyright @ 2018 Termedia \& Banach

\section{Abstract}

Introduction: The aim of the study was to analyse microbiological characteristics and clinical manifestations of cardiac device-related infective endocarditis (CDRIE) in cardiac resynchronization therapy (CRT) recipients, and to compare the diagnostic value of modified Duke (MDC) versus modified Duke lead criteria (MDLC; including to MDC local infection and pulmonary infection or embolism as major criteria).

Material and methods: The study population comprised 765 consecutive CRT patients from a high-volume, tertiary care centre from 2002 to 2015 . All patients were screened for CDRIE.

Results: During a median follow-up of 1692 days (range: 457-3067) 5.36\% of patients $(n=41)$ developed CDRIE, which was accompanied by CRT pocket infection in $17.1 \%(n=7)$ and recurrent pulmonary infection or pulmonary embolism in $29.3 \%(n=12)$. Fever was present in $95.1 \%$ of patients $(n=39)$, whereas blood cultures were positive in $65.9 \%(n=27)$. Staphylococcus was the most prevalent pathogen in $59.3 \%(n=16)$, Gram-negative bacteria in $25.9 \%(n=7)$. Transoesophageal echocardiography showed intracardiac vegetations in $73.2 \%$ of patients $(n=30)$. Non-different pathogen types with the most common methicillin-sensitive Staphylococcus aureus were observed for early versus late CDRIE (endocarditis $\leq 6$ vs. $>6$ months from CRT or other device-related procedure). All 3 inflammatory markers (C-reactive protein, white blood cells, procalcitonin) were normal in $4.9 \%$ of patients $(n=2)$. MDC versus MDLC indicated definite CDRIE in $48.8 \%$ versus $80.5 \%$, respectively $(p=0.003)$. Conclusions: Fever is the most common symptom of CRT-related CDRIE, and transoesophageal echocardiography allows vegetations to be visualised in nearly 3/4 of patients with CDRIE. Although the most common pathogens were Staphylococci, Gram-negative bacteria accounted for a quarter of CDRIE. Modified Duke lead criteria proved superior to MDC.

Key words: cardiac resynchronization therapy, endocarditis, heart failure, symptoms, signs.

\section{Introduction}

Cardiac device-related infective endocarditis (CDRIE) is a rare but one of the most serious complications that may occur in patients receiving

\author{
Corresponding author: \\ Ewa Jędrzejczyk-Patej \\ Department \\ of Cardiology \\ Congenital Heart \\ Diseases and \\ Electrotherapy \\ Silesian Centre for \\ Heart Diseases \\ 9 Skłodowskiej-Curie St \\ 41-800 Zabrze, Poland \\ Phone: +48 323733682 \\ E-mail: ewajczyk@op.pl
}


cardiac implantable electronic devices (CIEDs). Cardiac device-related infective endocarditis incidence and mortality have been reported at $0.1 \%$ to $5.1 \%$, and $0 \%$ to $35 \%$, respectively [1-4]. Patient's age, comorbidities, severity of heart failure (HF) or CIED type may play important roles in both CDRIE development and its prognosis [5, 6].

Cardiac resynchronization therapy (CRT) not only reduces heart failure (HF) symptoms and improves quality of life but also reduces mortality in HF patients with left-ventricular systolic dysfunction and electrical dyssynchrony [7-9]. Alas, compared to patients with pacemakers or cardioverter-defibrillators, CRT recipients seem to be at higher risk of CDRIE due to more advanced heart failure and higher complexity of CIEDs (and thus increased risk of device and lead-related complications) [3].

Prognosis in CDRIE is poor; thus early diagnosis and targeted therapy seem to be crucial for a positive outcome. However, diagnosing CDRIE is often difficult and complex. In addition, confirmation of CDRIE initiates "aggressive" treatment. Indeed, once CDRIE is evident, a combination antimicrobial treatment is routinely commenced, which may for example affect renal function, particularly in patients with chronic kidney disease. Also, complete device removal is indicated, which may be hazardous in pacemaker-dependent patients and those with severely decompensated HF, especially if inotropic support is also required. Modified Duke criteria (MDC) are routinely used to diagnose infective endocarditis. Although MDC are based on a combination of clinical, echocardiographic and biological findings, their diagnostic value in suspected CDRIE is limited [10]. Thus, various modifications thereof have been proposed, such as the modified Duke lead criteria (MDLC). Apart from classic major criteria such as positive blood cultures and imaging findings (vegetation, $a b-$ scess, etc.) and minor criteria used in MDC, MDLC also include local signs of infection and recurrent pulmonary infection or embolism as major criteria $[11,12]$. Nonetheless, the diagnostic role of MDLC has been assessed in only a few studies and never validated in CRT recipients [11-14].

The aim of our study was to analyse clinical manifestations and microbiological characteristics of CDRIE in CRT recipients, and to assess the applicability of modified versus modified Duke lead criteria in CRT patients.

\section{Material and methods}

\section{Study population}

The study design, factors influencing CDRIE development and outcomes in CRT recipients with CDRIE have been published previously [5]. In this analysis we sought to investigate the clinical picture of CRT-related endocarditis and to identify various pathogens associated with the disease development.

In brief, our population comprised consecutive HF patients with de novo or an up-grade CRT procedure, from a high volume tertiary care university centre, from May 2002 to March 2015. All patients met respective European Society of Cardiology (ESC) guideline recommendations for CRT implantation that were in effect at the time of the implant procedure. Thus, patients had symptomatic HF (the New York Heart Association [NYHA] class was II, III or IV despite optimal medical treatment); had to have wide QRS complexes; and had left ventricular ejection fraction (LVEF) $\leq 35 \%$. All subjects signed informed consent for the CRT procedure. The study complied with the Declaration of Helsinki.

\section{Implantation procedure and follow-up}

A standard implantation procedure was performed in all CRT recipients. A prophylactic single dose of cefazolin iv or clindamycin iv (in case of allergy to cephalosporins) was administered in all patients before the procedure. The electrodes were implanted endocardially via subclavian vein puncture or cut-down technique. The lateral or posterolateral vein was preferred as a target vein for left ventricular lead placement. The choice of electrode (unipolar vs. bipolar, passive vs. active fixation) and pocket type (subcutaneous vs. submuscular) was left to the discretion of the implanting physician.

Patients were followed from the time of CRT implantation until January 2017 or the patient's death. Scheduled visits were performed every 6 months, apart from the first two check-ups, which took place 1 week and 1 month after the implantation. Patient vital status was verified using data from the National Health Fund (insurer covering over $99 \%$ of the country population). Data from unscheduled visits, hospital records, telephone calls from patients and their relatives were also used in the analyses.

\section{Diagnosis and treatment of cardiac device-related infective endocarditis}

The study population was screened for CDRIE using modified Duke criteria [10]. Additional criteria such as signs of infection and pulmonary embolism or pulmonary infection i.e. modified Duke lead criteria (MDLC) were also taken into account [10-12].

Early CDRIE was defined as endocarditis that occurred $\leq 6$ months after CRT implantation or other device-related procedure, such as battery exchange, lead extraction, additional lead placement, etc. Late CDRIE was defined as endocarditis that occurred $>6$ months after the last procedure. 
Patients with CDRIE were treated in line with ESC guidelines. Empirical antimicrobial therapy was initiated once blood cultures were taken. Empirical antibiotic treatment was continued unless blood cultures were positive, in which case it was altered accordingly. Device removal was consid-

Table I. Baseline characteristics and laboratory findings

\begin{tabular}{|c|c|}
\hline Parameter & Value \\
\hline Age [years] & $59(48-75)$ \\
\hline Male & $33(80.5)$ \\
\hline Ischemic aetiology & $24(58.5)$ \\
\hline Primary prevention of SCD & $29(70.7)$ \\
\hline HA & $23(56.1)$ \\
\hline DM & $19(46.3)$ \\
\hline AF/AFL & $27(65.8)$ \\
\hline Early endocarditis & $21(51.2)$ \\
\hline \multicolumn{2}{|l|}{ Laboratory findings: } \\
\hline WBC $\left[\times 10^{3} / \mu \mathrm{l}\right]$ & $10.1(5.9-17.4)$ \\
\hline $\begin{array}{l}\text { Maximum WBC during index } \\
\text { hospitalization }\left[\times 10^{3} / \mu \mathrm{l}\right]\end{array}$ & $13.3(8.7-20.6)$ \\
\hline $\begin{array}{l}\text { hs-CRP at the moment of } \\
\text { CDRIE diagnosis }[\mathrm{mg} / \mathrm{l}]\end{array}$ & $54.6(5.05-224)$ \\
\hline $\begin{array}{l}\text { Maximum hs-CRP during } \\
\text { index hospitalization }[\mathrm{mg} / \mathrm{l}]\end{array}$ & $125.2(24-341.2)$ \\
\hline PCT [ng/ml] & $0.96(0.07-10)$ \\
\hline $\begin{array}{l}\text { Maximum PCT during index } \\
\text { hospitalization }[\mathrm{ng} / \mathrm{ml}]\end{array}$ & $1.56(0.3-11.1)$ \\
\hline $\begin{array}{l}\text { Creatinine at the moment of } \\
\text { CDRIE diagnosis }[\mu \mathrm{mol} / \mathrm{l}]\end{array}$ & $130(83-195)$ \\
\hline $\begin{array}{l}\text { Maximum creatinine during } \\
\text { index hospitalization }[\mu \mathrm{mol} / \mathrm{l}]\end{array}$ & $211(135-390)$ \\
\hline NT pro-BNP [pg/ml] & $7543(2667-34808)$ \\
\hline \multicolumn{2}{|l|}{ Symptoms and signs of CDRIE: } \\
\hline Fever & $39(95.1)$ \\
\hline Accompanied pocket infection & $7(17.1)$ \\
\hline Recurrent pulmonary infection & $9(21.9)$ \\
\hline Pulmonary embolism & $3(7.3)$ \\
\hline Electrical storm & $10(24.4)$ \\
\hline Positive blood cultures & $27(65.9)$ \\
\hline Vegetations in TEE & $30(73.2)$ \\
\hline
\end{tabular}

Variables are presented as number (\%) or median (range), as appropriate. AF - atrial fibrillation, AFL - atrial flutter, CDRIE - cardiac device related infective endocarditis, hs-CRP - highsensitivity C-reactive protein, DM - diabetes mellitus, HA - arterial hypertension, NT pro-BNP - N-terminal pro B-type natriuretic peptide, PCT - procalcitonin, SCD - sudden cardiac death, TEE transoesophageal echocardiography, WBC - white blood cells. ered in all patients, but the final decision was left to the discretion of the heart team.

\section{Statistical analysis}

The categorical variables were expressed as numbers and percentages, whereas continuous parameters were expressed as median \pm range. The $\chi^{2}$, Student's $t$ or Mann-Whitney $U$ test was used as appropriate to compare the groups.

All statistical analyses were performed using the software package Statistica (version 6.0, StatSoft Inc., Tulsa, OK, USA and version 10.0). A $p$-value of less than 0.05 was considered significant.

\section{Results}

\section{Study population}

The study population consisted of 765 consecutive, adult HF patients with CRT devices who were implanted in line with respective ESC guideline recommendations. There were $70.8 \%$ de novo CRT implantations, $13.7 \%$ up-grades from pacemakers and $15.5 \%$ up-grades from implantable cardioverter-defibrillators (ICD). Overall, CRT-D was implanted in $88.2 \%(n=675 / 765)$ patients and CRT-P in 90 (11.8\%).

Until January 2017, CDRIE was diagnosed in 41 patients (5.4\%). Baseline characteristics of CDRIE patients are presented in Table I. The most commonly used antibiotics for CDRIE were gentamycin (51.2\%, $n=21)$, cloxacillin $(29.3 \%, n=12)$ and vancomycin $(19.5 \%, n=8)$. The device was removed in 28 (68.3\%) patients and remained in situ in 13 (31.7\%) subjects. All-cause mortality of CDRIE patients was $51.2 \%(n=21 / 41)$; it was $39.3 \%(n=11 / 28)$ if the device was removed and $76.9 \%(n=10 / 13)$ if CRT removal was ultimately abandoned.

\section{Clinical manifestations of device-related infective endocarditis in CRT recipients}

\section{Fever}

Fever or recent fever (within 3 weeks preceding hospital admission) were the most common symptoms in CRT patients with CDRIE, at $95.1 \%$ $(n=39)$. Approximately a third of those $(31.7 \%$; $n=13)$ had a history of intermittent fever lasing $>1$ month (median: 4 months, range: $0.5-7$ ) with neither a proper diagnosis of CDRIE nor successful empirical antibiotic treatment.

\section{Local signs of infection}

Local device infection accompanied a CRT-related CDRIE in $17.1 \%(n=7)$ of patients. Of those, $3(7.3 \%)$ patients had a perforated pocket, whereas the remaining 4 patients had either superficial 
signs of inflammation (erythema), purulent drainage or pocket fluctuation. The pocket infection occurred in 1 patient $\leq 6$ months after CRT implantation and in 6 patients $>6$ months after the procedure.

\section{Pulmonary embolism and recurrent pulmonary infections}

Recurrent pulmonary infection was observed in $21.9 \%$ of patients $(n=9)$. None of them was successfully treated before CDRIE diagnosis. Chest computed tomography (CT) was performed in 6 patients and it showed pulmonary embolism in 3 .

\section{Electrical storm}

Recurrent, malignant ventricular arrhythmia with multiple adequate ICD therapies occurred in $24.4 \%(n=10)$ of patients, being not only the reason for urgent medical contact but also the first sign of CDRIE. Overall, 1 in 4 (23.3\%) electrical storms treated at our centre in the period from 2002 to 2017 was CDRIE-related.

\section{Blood cultures}

Positive blood cultures were present in $65.9 \%$ of patients $(n=27)$, whereas positive lead cultures were present in $41.5 \%$ (17 of 28 subjects) of those who had the device removed. The most common pathogens were Staphylococci (59.3\%; $n=16$ ); methicillin-sensitive Staphylococcus au- reus (MSSA) was identified in $37 \%$ of patients $(n=10)$, Enterococcus faecalis in $14.8 \%(n=4)$. Gram-negative bacteria were observed in $25.9 \%$ of patients $(n=7)$. The detailed distribution of pathogens is shown in Figure 1.

Pathogen profile was similar in early and late CDRIE. In early endocarditis $(51.2 \% ; n=21)$ negative cultures were present in $47.6 \%$ of patients $(n=10)$, whereas MSSA, MRSA, Staphylococcus epidermidis, Enterococcus faecalis, Klebsiella pneumoniae and Acinetobacter haemolyticus were identified in 5, 1, 1, 2, 1 and 1 patients, respectively. In late CDRIE (48.8\%; $n=20)$ the most common pathogen was MSSA ( $25 \% ; n=5)$; a non-different prevalence of MSSA infection compared with early CDRIE was observed. Fewer negative cultures were observed for late $(20 \% ; n=4)$ versus early CDRIE $(47.6 \% ; n=10 ; p=0.06)$.

\section{Laboratory findings}

The median C-reactive protein (CRP) level was above $5.00 \mathrm{mg} / \mathrm{l}$ at diagnosis in nearly all subjects $(90.2 \% ; n=37)$ as it was at least once above the upper range in all CDRIE patients during the index hospitalization. At the time of CDRIE diagnosis, white blood cells (WBC) were slightly elevated in $53.7 \%(n=22)$ of patients (median value of 10.1 $\times 10^{3} / \mu$ l (range: $5.9-17.41$ ); cut-off value of $10 \times$ $\left.10^{3} / \mu \mathrm{l}\right)$. Taking into account the whole hospital stay WBC were out of range in $78 \%(n=32)$ of patients. Procalcitonin (PCT) was > $0.5 \mathrm{ng} / \mathrm{ml}$ in

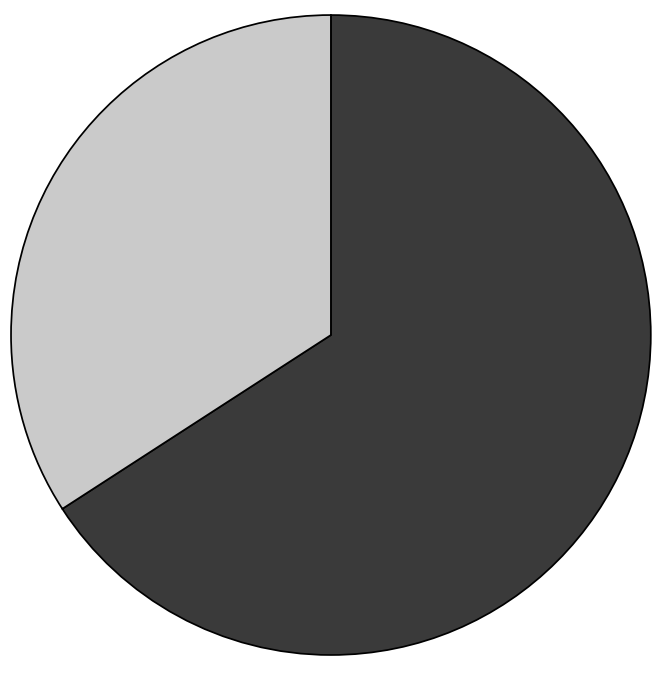

Positive cultures $66 \%$

Figure 1. Microbiological characteristics of CRT-related infective endocarditis

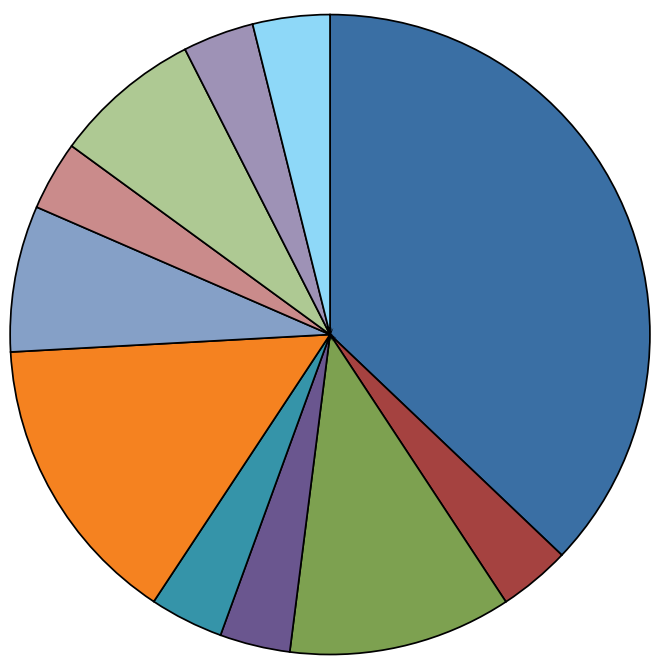

$\square$ MSSA $37 \% \quad \square$ MRSA $3.7 \%$
$\square$ S. lugdunesis $3.7 \%$

$\square$ Enterococcus faecalis $14.9 \%$

$\square$ S. epidermidis $11.1 \%$ $\square$ S. hominis $3.7 \%$ $\square$ Serratia marcescens $7.4 \%$ $\square$ K. pneumoniae $7.4 \%$ $\square$ E. coli $3.7 \%$

CRT - cardiac resynchronization therapy, E. coli - Escherichia coli, K. pneumoniae - Klebsiella pneumoniae, S. hominis Staphylococcus hominis, S. epidermidis - Staphylococcus epidermidis, S. lugdunensis - Staphylococcus lugdunensis, MRSA methicillin-resistant Staphylococcus aureus, MSSA - methicillin-sensitive Staphylococcus aureus. 
almost $50 \%$ of patients $(n=19 ; 46.3 \%)$ at CDRIE diagnosis, whereas the maximum PCT level during the index hospitalization was above the normal range in $58.5 \%$ of patients overall $(n=24)$. Two out of the three aforementioned inflammatory markers (CRP, WBC or CRP) were elevated in $43.9 \%(n=18)$ of patients, all 3 markers were increased in $36.6 \%$ ( $n=15)$ of patients, and only 2 (4.9\%) patients had all three markers within the normal range.

Three-quarters of subjects $(75.6 \% ; n=31)$ had an elevated creatinine level at hospital admission, the median value being $130 \mu \mathrm{mol} / \mathrm{l}(83-195)$ during CDRIE diagnosis. The laboratory data are shown in Table I.

\section{Echocardiography}

Echocardiography was performed in all patients with suspected CDRIE. Transthoracic echocardiography (TTE) showed intracardiac vegetations in $17.1 \%$ of patients $(n=7)$, whereas transoesophageal echocardiography (TEE) showed intracardiac vegetations in $73.2 \%(n=30 ; p<0.001)$. Location of vegetations was as follows: atrial lead $(29.3 \%$, $n=12)$, right ventricular lead $(34.1 \%, n=14)$, left ventricular lead $(21.9 \%, n=9)$, two or more electrodes $(12.2 \%, n=5)$. In $7(17.1 \%)$ patients vegetations on heart valves were also identified: tricuspid valve ( $n=5 ; 12.2 \%)$, mitral valve $(n=2 ; 4.9 \%)$. Other locations included: endocardium of the right ventricle $(n=1)$, intraatrial septum $(n=1)$, right atrial endocardium $(n=1)$, tricuspid valve cords $(n=1)$, mechanical mitral valve $(n=1)$ and tricuspid annulus $(n=1)$.

Vegetation prevalence was similar in early and late CDRIE (71.4\% vs. $75 \%$ respectively, $p=0.79$; Figure 2 B). Figure 2 illustrates CDRIE symptoms and signs.

A

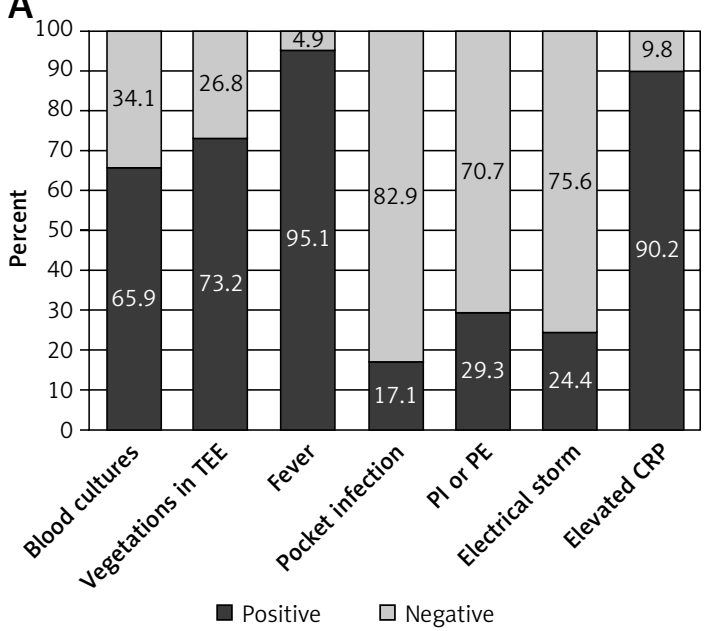

Modified Duke criteria and modified Duke lead criteria

The CDRIE diagnosis was established in all patients based on the modified Duke criteria. Two major Duke criteria were present in $48.8 \%$ of patients $(n=20)$, whereas also in $48.8 \%(n=20)$ one major and 1 minor criteria were fulfilled. In the remaining $2.4 \%(n=1) 3$ minor criteria were met. None of the patients met 5 minor criteria. Consequently, in line with MDC, a definite or possible CDRIE could be diagnosed in $48.8 \%$ and $51.2 \%$ of patients, respectively.

Taking into account to MDC one of the modified Duke lead criteria such as pocket infection as a major criterion, definite CDRIE could be diagnosed in $63.4 \%$ of patients $(n=26 ; p=0.18$ vs. MDC), whereas it could be suspected in $36.6 \%$ $(n=15)$. When to MDC other MDLC, i.e. pulmonary embolism or recurrent pulmonary infections, were included as additional major criteria, definite CDRIE were met in $70.7 \%$ of patients $(n=29 ; p=$ 0.04 vs. MDC). According to the MDLC (including local infection as well as pulmonary infection or embolism as major criteria), definite CDRIE could be diagnosed in $80.5 \%$ of patients $(n=33)$ and possible endocarditis in $19.5 \%(n=8)$.

Figure 3 presents the proportions of patients who can be diagnosed with CDRIE by using various diagnostic criteria, i.e. MDC, MDC + local infection, MDC + pulmonary infections/embolism, MDLC.

Modified Duke versus modified Duke lead criteria indicated definite CDRIE at 48.8 vs. $80.5 \%$, respectively ( $p=0.003$; Figure $3 \mathrm{~B}$ ).

\section{Discussion}

The main findings of our study are as follows: 1) fever was the most common symptom of

B

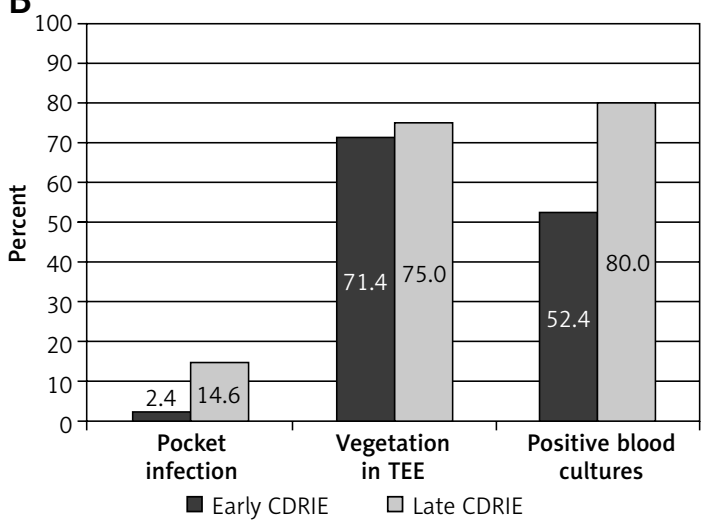

Figure 2. Clinical manifestations of CDRIE in patients undergoing CRT. A - Symptoms and signs of CDRIE in patients undergoing CRT, B - symptoms and signs of early versus late CDRIE

CDRIE - cardiac device-related infective endocarditis, CRP - high-sensitivity C-reactive protein, CRT-cardiac resynchronization therapy, PE - pulmonary embolism, PI - recurrent pulmonary infections, TEE - transoesophageal echocardiography. 
A

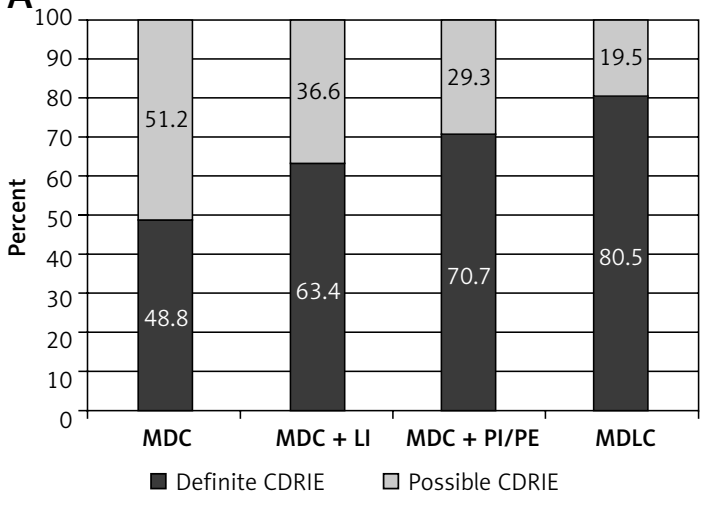

B
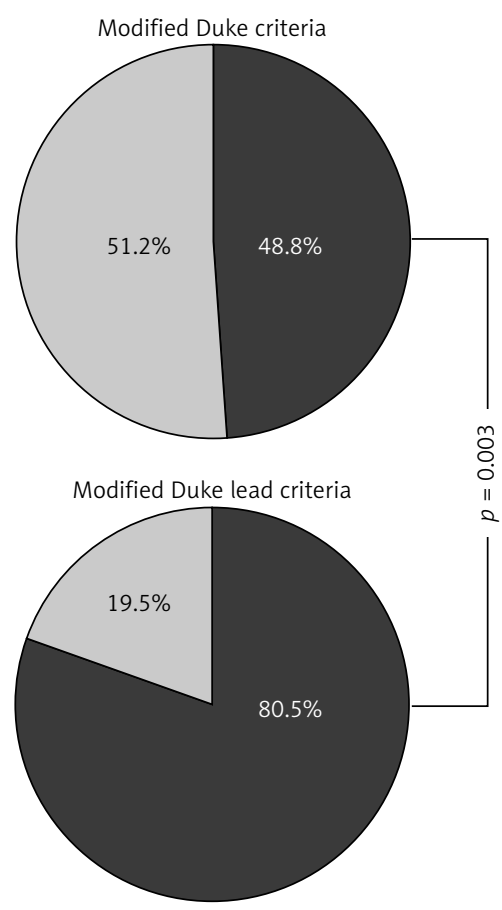

Figure 3. Diagnosis of CDRIE according to modified Duke criteria and their modifications. A - Definite and possible CDRIE according to modified Duke criteria and their modifications, B - comparison of Duke criteria - modified and modified lead criteria

CDRIE - cardiac device-related infective endocarditis, $L I$ - local infection, MDC - modified Duke criteria, MDLC - modified Duke lead criteria, PE - pulmonary embolism, $\mathrm{Pl}$ - recurrent pulmonary infections.

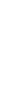

CRT-related CDRIE, at 95.1\%; 2) the most frequent CDRIE pathogens were Staphylococci or other Gram-positive bacteria, whereas over a third of blood cultures were negative; 3) TEE allowed vegetations to be shown in $72 \%$ of patients; 4 ) pocket infection accompanied CDRIE in 17\%; 5) recurrent pulmonary infection or pulmonary embolism was observed in $30 \%$ of patients with CDRIE; 6) CRP was high in $90 \%$ of CDRIE patients, whereas PCT was increased in nearly half of them; 7) modified Duke lead criteria proved superior to modified Duke criteria.

As we have reported previously, CDRIE develops in almost $5 \%$ of CRT recipients within 3.5 years after implantation and is associated with a very poor prognosis with mortality rates exceeding 65\% [5]. Thus, an early diagnosis of this life-threatening condition along with "aggressive" treatment seem to be crucial for a positive outcome. Unfortunately, there are no standardized methods to diagnose CDRIE. Duke criteria have never been validated in patients with CDRIE, and minor Duke criteria seem to have particularly low diagnostic value in this population. Beyond fever and predisposing heart disease, minor Duke criteria are not specific for right-sided endocarditis [11]. Thus, Duke criteria may be positive in CDRIE accompanied by left-sided infection but this subgroup constitutes less than $5 \%$ of CDRIE patients. Therefore, ESC guidelines suggested that pulmonary embolism or infections and local signs of infection should be considered as major criteria in the Duke system, and recent studies employed them as 'Modified Duke Lead Criteria' [12]. In our study, modified
Duke criteria and MDLC allowed definite endocarditis to be diagnosed in almost $50 \%$ and over $80 \%$ of patients, respectively. Indeed, inclusion of local signs of infection improved CDRIE detection rates by $14.6 \%$, while considering also pulmonary embolism and/or recurrent pulmonary infections further improved the diagnosis by $31.7 \%$. The diagnostic value of MDLC has been assessed in patients with various CIEDs, but not exclusively with CRT devices [15]. In our opinion, this may be of importance, as CRT recipients comprise a specific group of patients with advanced heart failure, numerous comorbidities, and often cachexia. Thus clinical manifestations of "CRT-related CDRIE" may differ from CDRIE in patients with less advanced heart failure and less complex cardiac devices.

Here, we provide important insights into clinically relevant factors affecting diagnosis and treatment of CRT-related CDRIE. First, we found that nearly all patients with CDRIE either had a history of recurrent fever or were febrile at CDRIE diagnosis. Although fever is not a CDRIE-specific symptom, our data indicate that less than $5 \%$ of patients with CDRIE did not have a fever. This is of importance, as contrary to perception (i.e. no symptoms or less well pronounced symptoms of infection due to anergy), patients with advanced/ terminal heart failure still do present with the most common symptom of underlying infection. In addition, almost all patients with CDRIE also had elevated inflammatory markers such as CRP, PCT and WBC, in $17 \%$ of cases despite previous antibiotic treatment. These simple, popular markers may be useful in CDRIE diagnosis, but they had 
relatively low specificity, and thus some new, cardiac specific markers might be more useful $[16,17]$. Nevertheless, our observations indicate that CDRIE should be suspected in every CRT patient who did not respond well to antibiotic therapy, has recurrent fever or has increased markers of infection.

Second, identification of CDRIE-specific pathogens is crucial for proper diagnosis and treatment. We found that a third of patients with CRT-related CDRIE had negative blood cultures, which considerably complicates decision making on treatment strategy. Nevertheless, that scenario might defer device removal, whereas leaving the device in situ in case of CDRIE was associated with significantly worse outcomes $[6,10]$. For those with positive blood cultures, the most common pathogens were Staphylococci (60\%) or other Gram-positive bacteria. The prevalence of Gram-negative pathogens was higher $(25 \%)$ in our study compared with previous reports $(1-17 \%)[4,18,19]$. The underlying disease, that is advanced heart failure, as well as the CRT device itself, may account for this difference. Indeed, infections caused by Gram-negative bacteria are more often healthcare-associated, occur in patients with an impaired immune system and are resistant to most commonly used antibiotics. Moreover, Gram-negative bacteria can survive on the surface of catheters or wounds. Thus, perhaps unsurprisingly, our microbiology findings do differ from previous reports which evaluated CDRIE in implantable devices other than CRT [20, 21]. To our knowledge, this study is the first to describe the epidemiology of causative pathogens specifically in CRT-related CDRIE. Nonetheless, in accord with previous reports [20,21], we did not observe any difference in bacteria prevalence regarding early versus late CDRIE, which may raise question about the rationale for differentiating early versus late infections.

Third, 3 in 4 patients in our study had vegetations that could be visualised using echocardiography $[12,19,22]$. Echocardiography is an established method for both qualifying patients for CRT therapy and assessing its response [23, 24]. Our findings show that echocardiography also plays a key role in diagnosing CRT-related CDRIE, particularly in the absence of positive blood cultures. As in previous reports $[25,26]$ we found that TEE was superior to TTE in detecting vegetations and thus should be performed in every patient with suspected CDRIE. Indeed, TEE allowed vegetations to be revealed not only on CRT leads or heart valves, but also within the right ventricle or even in the superior vena cava. Importantly, our data indicate that vegetations in CRT-related CDRIE can be visualised more frequently than in CDRIE associated with other, less complex implantable devices [2528]. Advanced HF, low EF, common comorbid dis- ease, in particular renal failure, are only examples of various important clinical factors that increase the probability of vegetation occurrence and thus may account for the observed difference $[27,28]$.

Our relatively small sample size along with a single centre experience and retrospective data analysis are limitations that have already been reported [5]. Consequently, more complex, adjusted multivariable modelling was not possible, and thus we provide herein crude event rates and descriptive data only. Nonetheless, CRT-related CDRIE is a rare condition and we present here data from over 750 consecutive patients with CRT devices implanted in a high volume, tertiary care university centre over the last 10 years.

In conclusion, fever is the most common symptom of CRT-related CDRIE and transoesophageal echocardiography allows intracardiac vegetations to be visualised in nearly $3 / 4$ of patients with CDRIE. Although the most common pathogens were Staphylococci, Gram-negative bacteria accounted for a quarter of CDRIE. Modified Duke lead criteria proved superior to modified Duke criteria.

\section{Conflict of interest}

Ewa Jędrzejczyk-Patej, Michał Mazurek, Oskar Kowalski, Agnieszka Liberska - consultant fees from Biotronik, Medtronic, St Jude Medical and Boston Scientific; Adam Sokal - travels reimbursement and consultant fees from Biotronik, Medtronic, St Jude Medical and Boston Scientific; lecturers from Medtronic, Boston Scientific and Impulse Dynamics; Radosław Lenarczyk -consultant fees and lecturers for Biotronik, Medtronic, St Jude Medical and Boston Scientific; Cordis Webster, Boeingher Ingelheim; Tomasz Podolecki - consultant fees from St Jude, Adamed, Abbott; Zbigniew Kalarus - received company sponsored speaker's bureau from Pfizer, Eli Lilly, Boehringer-Ingelheim, Abbott, Bayer; travel expenses to cardiology congresses from St. Jude Medical and Adamed; advisory committee: Boehringer-Ingelheim, Amgen, Astra Zeneca;

Other authors - no conflict of interests reported.

\section{References}

1. Baddour LM, Epstein AE, Erickson CC, et al. Update on cardiovascular implantable electronic device infections and their management: a scientific statement from the American Heart Association. Circulation 2010; 121: 458-77.

2. Cabell $\mathrm{CH}$, Heidenreich PA, Chu VH, et al. Increasing rates of cardiac device infections among Medicare beneficiaries: 1990-1999. Am Heart J 2004; 147: 582-6.

3. Hercé B, Nazeyrollas P, Lesaffre F, et al. Risk factors for infection of implantable cardiac devices: data from a registry of 2496 patients. Europace 2013; 15: 66-70.

4. Tarakji KG, Chan EJ, Cantillon DJ, et al. Cardiac implantable electronic device infections: presentation, man- 
agement, and patient outcomes. Hear Rhythm 2010; 7: 1043-7.

5. Jędrzejczyk-Patej E, Mazurek $M$, Kowalski O, et al. Device-related infective endocarditis in cardiac resynchronization therapy recipients - single center registry with over 2500 person-years follow up. Int J Cardiol 2017; 227: $18-24$

6. Deharo JC, Quatre A, Mancini J, et al. Long-term outcomes following infection of cardiac implantable electronic devices: a prospective matched cohort study. Heart 2012; 98: 724-31.

7. Tang ASL, Wells GA, Talajic M, et al. Cardiac-resynchronization therapy for mild-to-moderate heart failure. N Engl J Med 2010; 363: 2385-95.

8. Cleland JGF, Freemantle N, Erdmann E, et al. Long-term mortality with cardiac resynchronization therapy in the Cardiac Resynchronization-Heart Failure (CARE-HF) trial. Eur J Heart Fail 2012; 14: 628-34.

9. Chen A, Chen X, Shen Y, Li W. Modalities of ventricular pacing for cardiac resynchronization therapy in patients with heart failure: a meta-analysis and systematic review. Arch Med Sci 2017; 13: 1006-17.

10. Habib G, Lancellotti P, Antunes MJ, et al. 2015 ESC Guidelines for the management of infective endocarditis. Eur Heart J 2015; 36: 3075-128.

11. Polewczyk A, Janion M, Kutarski A. Cardiac device infections: definition, classification, differential diagnosis, and management. Pol Arch Med Wewn 2016; 126: 27583.

12. Polewczyk A, Janion M, Podlaski R, Kutarski A. Clinical manifestations of lead-dependent infective endocarditis: analysis of 414 cases. Eur J Clin Microbiol Infect Dis 2014; 33: 1601-8.

13. Klug D, Lacroix D, Savoye C, et al. Systemic infection related to endocarditis on pacemaker leads: clinical presentation and management. Circulation 1997; 95: 2098-107.

14. Sohail MR, Uslan DZ, Khan AH, et al. Infective endocarditis complicating permanent pacemaker and implant able cardioverter-defibrillator infection. Mayo Clin Proc 2008; 83: 46-53.

15. Polewczyk A, Jacheć W, Tomaszewski A, et al. Lead-related infective endocarditis: factors influencing early and long-term survival in patients undergoing transvenous lead extraction. Hear Rhythm 2017; 14: 43-9.

16. Ptaszynska-Kopczynska K, Szpakowicz A, Marcinkiewicz-Siemion M, et al. Interleukin- 6 signaling in patients with chronic heart failure treated with cardiac resynchronization therapy. Arch Med Sci 2017; 13: 1069-77.

17. Plenz G, Song ZF, Tjan TD, et al. Activation of the cardiac interleukin-6 system in advanced heart failure. Eur J Heart Fail 2001; 3: 415-21.

18. Bongiorni MG, Tascini C, Tagliaferri E, et al. Microbiology of cardiac implantable electronic device infections. Europace 2012; 14: 1334-9.

19. Sandoe JAT, Barlow G, Chambers JB, et al. Guidelines for the diagnosis, prevention and management of implantable cardiac electronic device infection. Report of a joint Working Party project on behalf of the British Society for Antimicrobial Chemotherapy (BSAC, host organization), British Heart Rhythm Society (BHRS), British Cardiovascular Society (BCS), British Heart Valve Society (BHVS) and British Society for Echocardiography (BSE). J Antimicrob Chemother 2015; 70: 325-59.

20. Sohail MR, Hussain S, Le KY, et al. Risk factors associated with early- versus late-onset implantable cardiovert er-defibrillator infections. J Interv Card Electrophysiol 2011; 31: 171-83.

21. Greenspon AJ, Prutkin JM, Sohail MR, et al. Timing of the most recent device procedure influences the clinical outcome of lead-associated endocarditis results of the MEDIC (Multicenter Electrophysiologic Device Infection Cohort). J Am Coll Cardiol 2012; 59: 681-7.

22. Greenspon AJ, Rhim ES, Mark G, Desimone J, Ho RT. Lead-associated endocarditis: the important role of methicillin-resistant Staphylococcus aureus. Pacing Clin Electrophysiol 2008; 31: 548-53.

23. Gąsior Z, Płońska-Gościniak E, Kułach A, et al. Impact of septal flash and left ventricle contractile reserve on positive remodeling during 1 year cardiac resynchronization therapy: the multicenter ViaCRT study. Arch Med Sci 2016; 12: 349-52.

24. Linde C, Leclercq C, Rex S, et al. Long-term benefits of biventricular pacing in congestive heart failure: results from the MUltisite STimulation in cardiomyopathy (MUSTIC) study. J Am Coll Cardiol 2002; 40: 111-8.

25. Vilacosta I, Sarriá C, San Román JA, et al. Usefulness of transesophageal echocardiography for diagnosis of infected transvenous permanent pacemakers. Circulation 1994; 89: 2684-7.

26. Victor F, De Place C, Camus C, et al. Pacemaker lead infection: echocardiographic features, management, and outcome. Heart 1999; 81: 82-7.

27. Golzio PG, Fanelli AL, Vinci $M$, et al. Lead vegetations in patients with local and systemic cardiac device infections: prevalence, risk factors, and therapeutic effects. Europace 2013; 15: 89-100.

28. Greenspon AJ, Le KY, Prutkin JM, et al. Influence of vegetation size on the clinical presentation and outcome of lead-associated endocarditis: results from the MEDIC registry. JACC Cardiovasc Imaging 2014; 7: 541-9. 